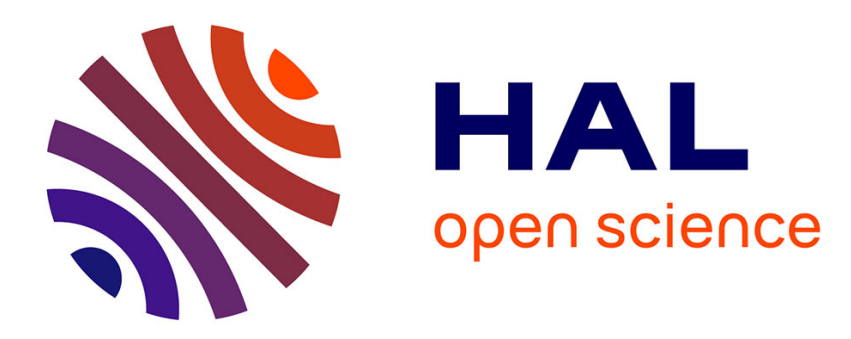

\title{
Nontrivial effects of high-frequency excitation for strongly damped mechanical systems
}

Alexander Fidlin, Jon Juel Thomsen

\section{To cite this version:}

Alexander Fidlin, Jon Juel Thomsen. Nontrivial effects of high-frequency excitation for strongly damped mechanical systems. International Journal of Non-Linear Mechanics, 2008, 43 (7), pp.569. 10.1016/j.ijnonlinmec.2008.02.002 . hal-00501779

\section{HAL Id: hal-00501779 https://hal.science/hal-00501779}

Submitted on 12 Jul 2010

HAL is a multi-disciplinary open access archive for the deposit and dissemination of scientific research documents, whether they are published or not. The documents may come from teaching and research institutions in France or abroad, or from public or private research centers.
L'archive ouverte pluridisciplinaire HAL, est destinée au dépôt et à la diffusion de documents scientifiques de niveau recherche, publiés ou non, émanant des établissements d'enseignement et de recherche français ou étrangers, des laboratoires publics ou privés. 


\section{Author's Accepted Manuscript}

Nontrivial effects of high-frequency excitation for strongly damped mechanical systems

Alexander Fidlin, Jon Juel Thomsen

PII: S0020-7462(08)00037-1

DOI: doi:10.1016/j.ijnonlinmec.2008.02.002

Reference: NLM 1449

To appear in: International Journal of Non-

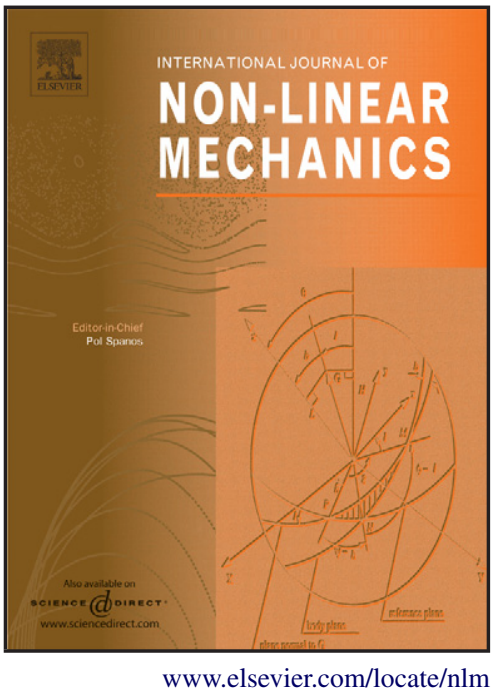
Linear Mechanics

Received date: 14 September 2007

Revised date: 4 February 2008

Accepted date: 5 February 2008

Cite this article as: Alexander Fidlin and Jon Juel Thomsen, Nontrivial effects of highfrequency excitation for strongly damped mechanical systems, International Journal of Non-Linear Mechanics (2008), doi:10.1016/j.ijnonlinmec.2008.02.002

This is a PDF file of an unedited manuscript that has been accepted for publication. As a service to our customers we are providing this early version of the manuscript. The manuscript will undergo copyediting, typesetting, and review of the resulting galley proof before it is published in its final citable form. Please note that during the production process errors may be discovered which could affect the content, and all legal disclaimers that apply to the journal pertain. 


\title{
Nontrivial effects of high-frequency excitation for strongly damped mechanical systems
}

\author{
A lexander Fidlin ${ }^{1}$ and J on Juel Thomsen ${ }^{2}$ \\ ${ }^{1}$ LuK GmbH \& Co. oHG, Industriestr. 3, D-77815, Buehl, Germany \\ ${ }^{2}$ Department of Mechanical Engineering, Solid Mechanics, Technical University \\ of Denmark, Building 404, DK-2800 Lyngby, Denmark
}

\begin{abstract}
Some nontrivial effects are investigated, which can occur if strongly damped mechanical systems are subjected to strong high-frequency (HF) excitation. The main result is a theoretical prediction, supported by numerical simulation, that for such systems the (quasi-) equilibrium states can change substantially with the level of damping. For example, a strongly damped pendulum, with a hinge vibrated at high frequency along an elliptical path with horizontal or vertical axis, will line up along a line offset from the vertical; the offset vanishes for very light or very strong damping, attaining a maximum that can be substantial (depending on the strength of the HF excitation) for finite values of the damping. The analysis is focused on the differences between the classic results for weakly damped systems, and new effects for which the strong damping terms are responsible. The analysis is based on a slightly modified averaging technique, and includes an elementary example of an elliptically excited pendulum for illustration, al ongside with a generalization to a broader class of strongly damped dynamical systems with $\mathrm{HF}$ excitation. A s an application example, the nontrivial behavior of a classical optimally controlled nonlinear system is investigated, illustrating how HF excitation may cause the controller to leave the system in an unexpected equilibrium state, quite different from the setpoint. The effects can be interesting for specialists in control of mechanical systems and structures. How ever the obtained results are more general. Similar effects could be expected first of all for microsystems where damping forces are typically dominating over inertia forces.
\end{abstract}

Keywords: high-frequency excitation, strong damping, effective properties, control, averaging

\section{Introduction}

Nontrivial effects of high-frequency (HF) excitation on mechanical systems have been investigated intensively in the last decade. The monograph by Blekhman [1] is the main reference in this area. The main effects of HF excitation in mechanical systems can be classified into three groups [2,3]:

- Stiffening is the ability of HF excitation to increase (or decrease) the effective stiffness of the mechanical system, with respect to slow motions; one of the consequences of this is the ability to stabilize or destabilize equilibria through changing the sign of the effective stiffness.

- Biasing is the ability of HF excitation to attract the mechanical system to a new steady state, e.g., a quasi-equilibrium or a steady state velocity (like in all cases of vibration induced transportation).

- Smoothening is the ability of HF excitation to smoothen nonlinearities, on the average; especially important is this effect for systems with dry friction, where HF dither is often used to reduce the effective hysteretic behavior with respect to slow motions.

Different mathematical approaches can be used to obtain the equations governing the slow motions of a mechanical system under HF excitation. Standard averaging $[4,5]$ or multiple scales analysis [6] are the general ones, whereas the method of the direct separation of motions [1] is highly specialized and effective in this particular area [7,8]. In [3,5] these 
techniques are briefly compared, and shown to give identical or very similar results.

However, the main attention in these publications is paid to weakly damped systems which are usual in mechanical applications. In that case the strong HF excitation terms are balanced with large inertia forces (i.e. acceleration terms which are proportional to squared frequency). There are at least two application areas where the situation is significantly different: First, it is quite usual to apply strong damping to control systems. The reason for that is the objective to avoid unnecessary oscillations, and to lead the controlled system to a given position as soon as possible. HF vibrations are sometimes used in control systems, e.g. to smoothen the effective dry friction $[9,10]$. However, in the presence of strong damping terms, a strong HF excitation may prevent correct operation of the control system, e.g. the controller may leave the system at an equilibrium that is shifted away from the desired setpoint [11]. A nother and perhaps even more important area is microsystems. Inertia terms are generally proportional to the mass of the system, i.e. to the cube of its linear dimension, while damping forces are usually applied to the surface of the body and thus proportional to the square of the linear dimension. Decreasing linear dimension therefore increases the importance of the damping terms.

This paper investigates nontrivial effects that may occur if a strongly damped mechanical system is subjected to strong HF excitation. The analysis is focused on the differences between the classic results well known for weakly damped systems, and new effects for which the strong damping terms are responsible. A classic example of a weakly damped pendulum subjected to elliptic vibrations of its suspension point is considered in Section 2, and compared to the same system with strong damping. These results are generalized in Section 3. Finally, as a more complicated example showing application of the general results, a classical nonlinear system under optimal control (an up-pointing pendulum on a cart) and its (mis)- behavior under HF excitation is analyzed in section 4.

\section{Illustrative example: Pendulum with elliptic HF excitation}

Consider the pendulum in Fig. 1 having length $l$ and mass $m$ in gravity $g$, whose suspension point is excited harmonically at frequency $\tilde{\omega}=\omega \sqrt{g / l}$, and horizontal and vertical displacement amplitudes $a_{h} l$ and $a_{v} l$, respectively, with a relative phase $\gamma$. The equation of motion is, in nondimensional form:

$$
\ddot{\theta}+2 \beta \dot{\theta}+\sin \theta=a_{v} \omega^{2} \sin \omega t \sin \theta+a_{h} \omega^{2} \sin (\omega t+\gamma) \cos \theta
$$

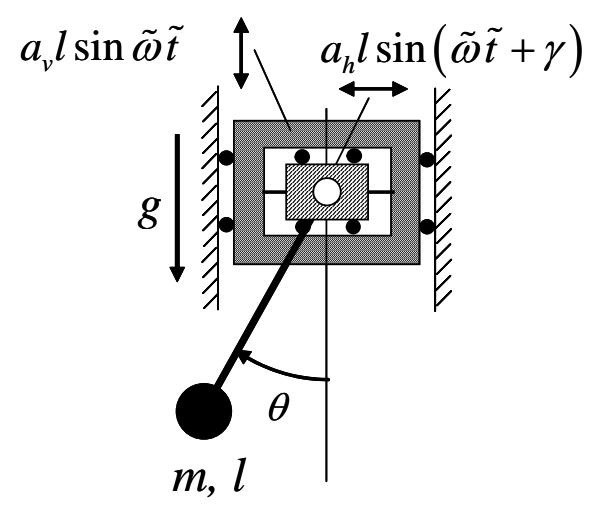

Fig. 1. M odel of the excited pendulum. 
where dots denote differentiation with respect to nondimensional time $t=\tilde{t} \sqrt{g / l}$ and linear damping with coefficient $\beta$ has been added. The chosen excitation form means the suspension point moves along an ellipse, whose orientation is determined by $a_{h} / a_{v}$ and by $\gamma$.

We suppose here, following the standard analysis [1-5], that the excitation frequency is much higher than the pendulum natural frequency, and that the excitation displacement amplitudes are small, so that the velocity amplitudes are of order unity, i.e.:

$$
\begin{aligned}
& \omega^{-1} \square 1 ; \quad a_{v}=O\left(\omega^{-1}\right) ; \quad a_{h}=O\left(\omega^{-1}\right) \\
& a_{v} \omega=A_{v}=O(1) ; \quad a_{h} \omega=A_{h}=O(1) .
\end{aligned}
$$

\subsection{Case of weak damping}

First we assume that the damping is not large, i.e. $\beta=O(1)$. Introducing a fast time $\tau=\omega t$ as the new independent variable, and $\rho=\dot{\theta}$ as a new dependent variable, we convert (1) to the following system of two first-order differential equations:

$$
\begin{aligned}
& \theta^{\prime}=\varepsilon \rho \\
& \rho^{\prime}=-2 \varepsilon \beta \rho-\varepsilon \sin \theta+A_{v} \sin \tau \sin \theta+A_{h} \sin (\tau+\gamma) \cos \theta
\end{aligned}
$$

where $\varepsilon=1 / \omega \square 1$, and ()$^{\prime}=d / d \tau$.

The non-small terms in (3) can be eliminated if we apply the following transformation (based on the solution to the unperturbed problem, i.e. $\varepsilon=0$ in (3)):

$$
\rho=\sigma-A_{r} \cos \tau \sin \theta-A_{h} \cos (\tau+\gamma) \cos \theta .
$$

The resulting equations for $\theta(\tau)$ and $o(\tau)$ are then in the standard form for averaging $[4,5]$. Considering the first-order approximation, and returning to the slow time $t$, we obtain the following equation governing the slow motions of the elliptically excited pendulum (index 1 here and further denotes a first-order averaging approximation):

$$
\ddot{\theta}_{1}+2 \beta \dot{\theta}_{1}+\sin \theta_{1}=-\frac{1}{4}\left(A_{\nu}^{2}-A_{h}^{2}\right) \sin 2 \theta_{1}-\frac{1}{2} A_{\nu} A_{h} \cos \gamma \cos 2 \theta_{1} .
$$

This equation explains the well known effects of $\mathrm{HF}$ excitation for pendulums [1-3,5]:

- Stabilization of the up-pointing equilibrium by pure vertical excitation $\left(A_{h}=0\right)$.

- Destabilization of the down-pointing equilibrium by pure horizontal excitation $\left(A_{v}=0\right)$.

- B iasing of the pendulum towards the main excitation axis in case of elliptic excitation $\left(A_{h} A_{v} \neq 0\right)$.

The last effect can be visualized if we introduce the corresponding effective (i.e. average) potential for (5):

$$
\Pi=1-\cos \theta_{1}+\frac{1}{8}\left(A_{r}^{2}-A_{h}^{2}\right)\left(1-\cos 2 \theta_{1}\right)+\frac{1}{4} A_{r} A_{h} \cos \gamma \sin 2 \theta_{1} .
$$

which is depicted in Fig. 2 for different values of the phase difference $\gamma$. The minima of the potential function correspond to stable quasi-equilibria of the elliptically excited pendulum. Biasing occurs if, in the presence of HF excitation, these equilibria are shifted away from the unexcited equilibria $\theta=j \pi, j \in \mathrm{Z}$. The bias is maximal for $\gamma=0$ or $\gamma=\pi$. For estimating the bias offset from the down-pointing equilibrium, we can linearise (5) in the vicinity of $\theta=0$, and obtain an approximate expression for the new stationary equilibrium in presence of $\mathrm{HF}$ excitation: 


$$
\theta_{1 s t}=-\frac{A_{v} A_{h} \cos \gamma}{2+A_{v}^{2}-A_{h}^{2}} .
$$

Note firstly that there is no bias if $\gamma= \pm \pi / 2$, i.e. if the excitation ellipse has a horizontal or vertical axis, and secondly that the stationary bias does not depend on the damping coefficient $\beta$.

\subsection{Case of strong damping}

A gain we investigate the system (1), but assuming instead the damping to be strong, i.e.

$$
2 \beta=k \omega>>1, k=O(1),
$$

so that instead of (3) one has:

$$
\begin{aligned}
& \theta^{\prime}=\varepsilon \rho \\
& \rho^{\prime}=-k \rho-\varepsilon \sin \theta+A_{\gamma} \sin \tau \sin \theta+A_{h} \sin (\tau+\gamma) \cos \theta
\end{aligned}
$$

Now we eliminate the non-small oscillating terms, though the strong damping term will be still present. This can be achieved by the following transformation, from $\rho$ to a new variable $q$, based on the particular solution of the unperturbed system (i.e. (9) with $\varepsilon=0$ ):

$$
\begin{aligned}
\rho= & q+F_{s}(\theta) \sin \tau+F_{c}(\theta) \cos \tau, \text { where } \\
F_{s}(\theta) & =\frac{k A_{v} \sin \theta+A_{h}(\sin \gamma+k \cos \gamma) \cos \theta}{1+k^{2}} \\
F_{c}(\theta) & =-\frac{A_{v} \sin \theta-A_{h}(k \sin \gamma-\cos \gamma) \cos \theta}{1+k^{2}} .
\end{aligned}
$$

A pplying this transformation to (9) we obtain:

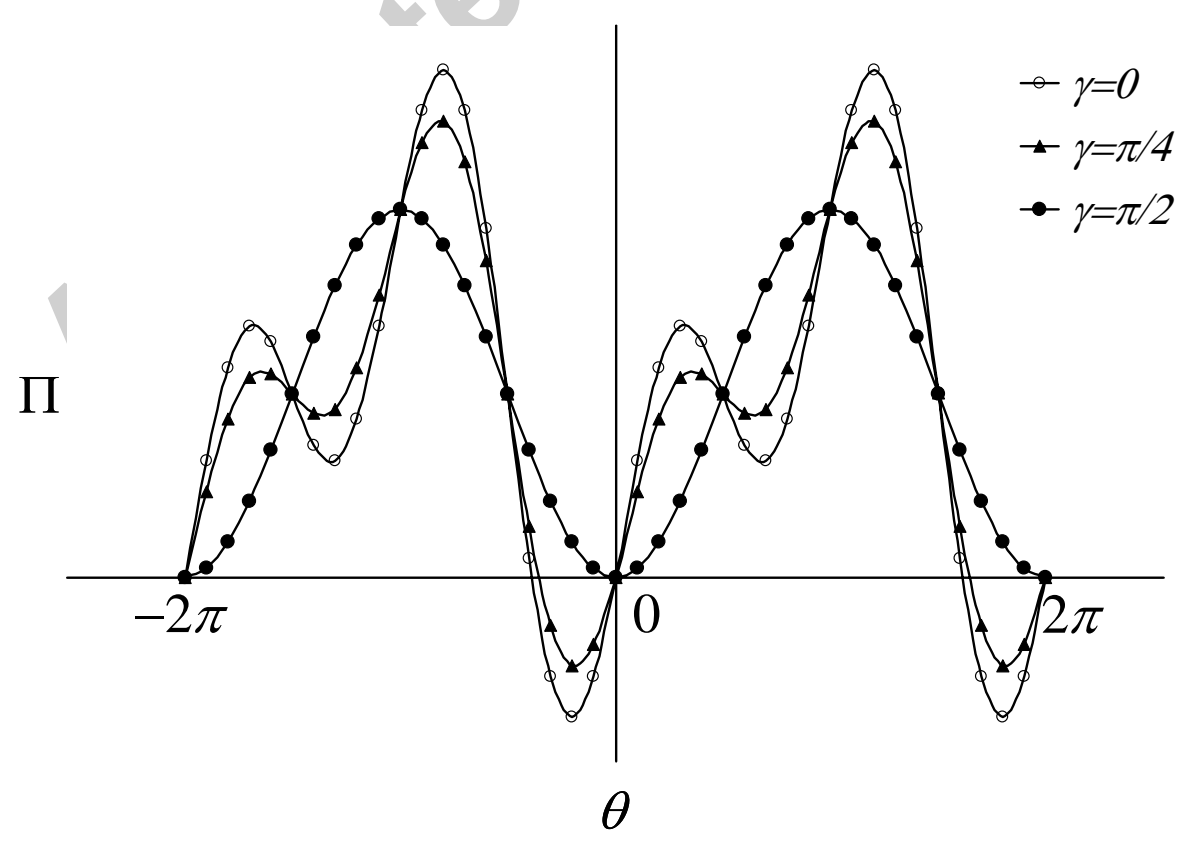

Fig. 2. Potential function for the slow motions of the elliptically excited pendulum. 


$$
\begin{aligned}
\theta^{\prime}= & \varepsilon q+\varepsilon F_{s}(\theta) \sin \tau+\varepsilon F_{c}(\theta) \cos \tau \\
q^{\prime}= & -k q-\varepsilon \sin \theta-\varepsilon\left(F_{s}^{\prime}(\theta) \sin \tau+F_{c}^{\prime}(\theta) \cos \tau\right)\left(q+F_{s}(\theta) \sin \tau+F_{c}(\theta) \cos \tau\right), \\
& F_{s}^{\prime}(\theta)=\frac{k A_{v} \cos \theta-A_{h}(\sin \gamma+k \cos \gamma) \sin \theta}{1+k^{2}} \\
& F_{c}^{\prime}(\theta)=-\frac{A_{v} \cos \theta+A_{h}(k \sin \gamma-\cos \gamma) \sin \theta}{1+k^{2}}
\end{aligned}
$$

where primes on $F_{s}$ and $F_{c}$ indicate derivatives with respect to $\theta$. The structure of these equations is typical for systems with strong damping. The procedures for the corresponding asymptotic analysis (including mathematical proof and error estimation) can be found in $[5,12]$. The first-order approximation (direct averaging of the right hand sides of the two first equations in (11)) is:

$$
\begin{aligned}
& \theta_{1}^{\prime}=0 \\
& q_{1}^{\prime}=-k q_{1}-\varepsilon \sin \theta-\frac{1}{2} \varepsilon\left(F_{s}^{\prime}(\theta) F_{s}(\theta)+F_{c}^{\prime}(\theta) F_{c}(\theta)\right),
\end{aligned}
$$

which, however, gives insufficient information about the system's dynamics: It fails in predicting any evolution of $\theta$, and demonstrates only the dominance of the damping term. Thus the second-order approximation has to be considered. The appropriate general results for this can be formulated in the following theorem [5]:

Consider a system with a slow master variable $x$ and a strongly damped slave variable $y$ :

$$
\begin{aligned}
& x^{\prime}=\varepsilon X(x, y, t) \\
& y^{\prime}=-k y+\varepsilon Y(x, y, t) \\
& x(0)=x_{0} ; y(0)=y_{0}
\end{aligned}
$$

where $k>0$ and $0<\varepsilon \square 1$, alongside with the following second-order approximation:

$$
\begin{aligned}
& \xi^{\prime}=\varepsilon \Xi_{1}(\xi)+\varepsilon^{2} \Xi_{2}(\xi) \\
& \eta^{\prime}=-k \eta+\varepsilon Y(\xi, 0, t) \\
& \xi(0)=x_{0} ; \eta(0)=y_{0} ; \\
& \Xi_{1}(\xi)=\langle X(\xi, 0, t)\rangle \\
& \Xi_{2}(\xi)=\left\langle\left.\frac{\partial X}{\partial x}\right|_{y=0} u+\left.\frac{1}{k} \frac{\partial X}{\partial y}\right|_{y=0} Y(\xi, 0, t)\right\rangle ; u=\int_{0}^{t}\left(X(\xi, 0, \tau)-\Xi_{1}(\xi)\right) d \tau,
\end{aligned}
$$

where \langle\rangle denotes averaging with respect to time $t$. Under the usual requirements $[4,5]$ on continuity of the right hand sides in (13), the solutions of the systems (13) and (14) are close, with a residual error $O\left(\varepsilon^{2}\right)$ on an asymptotically long time interval after a short transient process at the very beginning, i.e.:

$$
\|x-\xi\|=O\left(\varepsilon^{2}\right), \quad\|y-\eta\|=O\left(\varepsilon^{2}\right), \quad O\left(\frac{1}{\ln \varepsilon}\right)<t<O\left(\frac{1}{\varepsilon}\right) .
$$

A pplying this theorem to the transformed pendulum system (11), we calculate the necessary averages: 


$$
\begin{aligned}
& X=q+F_{s}(\theta) \sin \tau+F_{c}(\theta) \cos \tau \\
& \Xi_{1}=0 ; \quad u=-F_{s} \cos \tau+F_{c} \sin \tau \\
& Y(\theta, 0, \tau)=-\sin \theta-\left(F_{s}^{\prime}(\theta) \sin \tau+F_{c}^{\prime}(\theta) \cos \tau\right)\left(q+F_{s}(\theta) \sin \tau+F_{c}(\theta) \cos \tau\right) \\
& \left.\frac{\partial X}{\partial \theta}\right|_{q=0}=F_{s}^{\prime} \sin \tau+F_{c}^{\prime} \cos \tau ;\left.\quad \frac{\partial X}{\partial q}\right|_{q=0}=1 \\
& \Xi_{2}=-\frac{1}{2} \frac{A_{v} A_{h} \sin \gamma}{1+k^{2}}-\frac{1}{k} \sin \theta-\frac{1}{2 k}\left(\frac{1}{2} \frac{A_{v}^{2}-A_{h}^{2}}{1+k^{2}} \sin 2 \theta-\frac{A_{v} A_{h} \cos \gamma}{1+k^{2}} \cos 2 \theta\right),
\end{aligned}
$$

and obtain the second-order approximation:

$$
\begin{aligned}
& \theta_{2}^{\prime}=-\varepsilon^{2}\left(\frac{1}{k} \sin \theta_{2}+\frac{1}{2} \frac{A_{v} A_{h} \sin \gamma}{1+k^{2}}+\frac{1}{4 k} \frac{A_{v}^{2}-A_{h}^{2}}{1+k^{2}} \sin 2 \theta_{2}-\frac{A_{v} A_{h} \cos \gamma}{2 k\left(1+k^{2}\right)} \cos 2 \theta_{2}\right) \\
& q_{2}^{\prime}=-k q_{2}-\varepsilon \sin \theta_{2}-\varepsilon\left(F_{s}^{\prime}\left(\theta_{2}\right) \sin \tau+F_{c}^{\prime}\left(\theta_{2}\right) \cos \tau\right)\left(F_{s}\left(\theta_{2}\right) \sin \tau+F_{c}\left(\theta_{2}\right) \cos \tau\right) .
\end{aligned}
$$

The first equation is the important one, describing the slow dynamics of the strongly damped pendulum by a first-order differential equation. To estimate the bias of the downpointing equilibrium, we linearise (17) near $\theta=0$, and obtain an approximate expression for the new equilibrium in the presence of $\mathrm{HF}$ excitation:

$$
\theta_{2 s t}=\frac{A_{v} A_{h}(k \sin \gamma-\cos \gamma)}{2\left(1+k^{2}\right)+A_{t}^{2}-A_{h}^{2}} .
$$

which approaches the solution (7) for the weakly damped pendulum as the damping coefficient $k \rightarrow 0$.

The structure of the solutions (7) and (18) are similar: both vertical and horizontal excitation must be present to bias the pendulum. However, there are at least two significant differences. Firstly, the variation of the stationary bias $\theta_{2 n d}$ with the phase $\gamma$ is more complex now, with the phase difference generating the strongest bias (largest $\left.\left|\theta_{2 n d}\right|\right)$ being dependent on

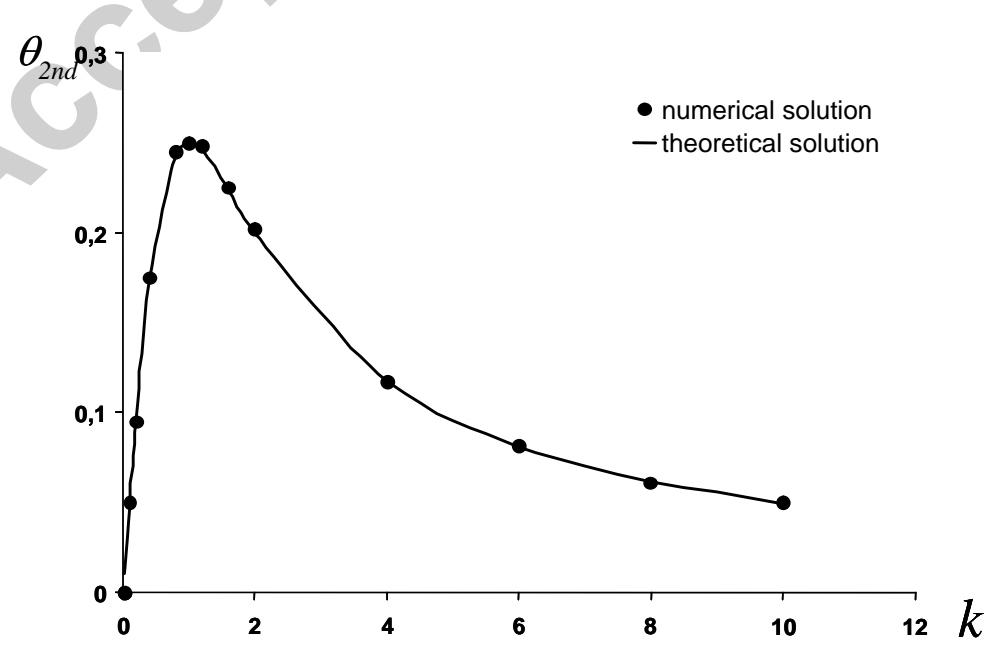

Fig. 3. Variation of stationary pendulum equilibrium bias $\theta_{2 \text { nd }}$ with damping $k$, for a maximizing value of phase $\gamma$. (Parameters: $A_{v}=1, A_{h}=1, \omega=50, \gamma=-\pi / 2$.) 
damping. Secondly, the bias itself depends strongly on the damping coefficient: For the weakly damped pendulum, there is no bias in the case of elliptical excitation with horizontal or vertical major axes $(\gamma= \pm \pi / 2)$. The strongly damped pendulum, by contrast, is biased in that case, with a maximum bias for damping $k=1$. This is illustrated in Fig. 3, displaying the approximate prediction (18) for parameters as given in the legend; as appears the results agree with results of numerical simulation of the full system (1). Fig. 4 shows how the bias depends on the phase difference for the same parameter values and the maximizing value $k=1$; as appears phase control can be used to shift the biased equilibrium continuously through a range of negative and positive angles.

Note that (18) is based on linearization, assuming small $\theta_{2 s}$. Thus, the seeming possibility of infinite bias as the denominator approaches zero is spurious; the solution of the full system (17) remains limited.

Returning to dimensional form we can rewrite (18) as follows:

$$
\theta_{2 s t}=\frac{a_{v} a_{h} \tilde{\omega}^{2} l\left(\frac{2 \beta}{\tilde{\omega}} \sqrt{\frac{g}{l}} \sin \gamma-\cos \gamma\right)}{\left(a_{v}{ }^{2}-a_{h}{ }^{2}\right) \tilde{\omega}^{2} l+2 g\left(1+\frac{4 \beta^{2} g}{\tilde{\omega}^{2} l}\right)}
$$

This form of the solution demonstrates explicitly that if the vibration acceleration is dominant compared to gravity, i.e. $\tilde{\omega}^{2}>>g / l$, then the stationary tilt of the damped pendulum depends on the phase difference between the vertical and the horizontal excitations in the same way as it does in case of the weak damping (cf. (7)).

\section{Generalization}

Consider the following system:

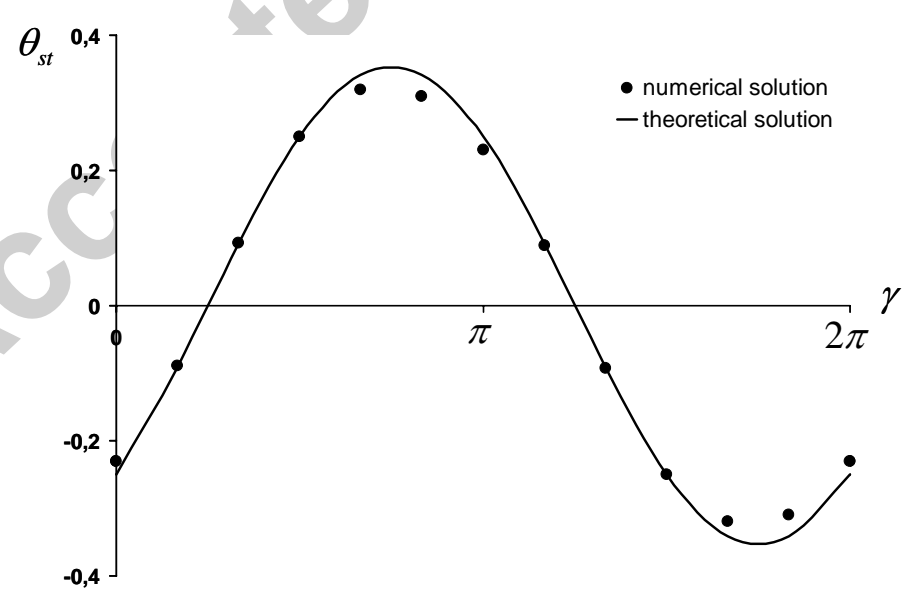

Fig. 4. Variation of pendulum bias $\theta_{\text {st }}$ with phase difference $\gamma_{\text {. }}$ (Parameters as for Fig. 3, and $k=1$.) 


$$
\begin{aligned}
& \mathbf{x}^{\prime}=\varepsilon \mathbf{h}(\mathbf{y})+\varepsilon \mathbf{X}(\mathbf{x}, \tau) \\
& \mathbf{y}^{\prime}=-\mathbf{K y}+\varepsilon \mathbf{G}(\mathbf{x}, \tau) \\
& \mathbf{h}(\mathbf{0})=\mathbf{0}, \quad \varepsilon<<1 \\
& \mathbf{K}(\mathbf{x})=\operatorname{diag}\left[k_{1}(\mathbf{x}) \quad k_{2}(\mathbf{x}) \quad \cdots \quad k_{m}(\mathbf{x})\right], k_{i}(\mathbf{x})>0, i=1, \ldots, m .
\end{aligned}
$$

where the vector functions $\mathbf{h}, \mathbf{X}, \mathbf{G}$ and the diagonal damping matrix $\mathbf{K}$ are bounded and Lipschitz-continuous in $\mathbf{x}$ and $\mathbf{y}$, and $\mathbf{X}$ and $\mathbf{G}$ are $2 \pi$-periodic in the fast time $\tau$.

A pplying the same approach as above we obtain the second order approximation:

$$
\begin{aligned}
& \xi^{\prime}=\varepsilon \boldsymbol{\Xi}_{1}(\xi)+\varepsilon^{2} \Xi_{2}(\xi) \\
& \eta^{\prime}=-\mathbf{K}(\xi) \boldsymbol{\eta}+\varepsilon \mathbf{G}(\boldsymbol{\eta}, \tau)
\end{aligned}
$$

Note that the steady state solution for $\mathbf{y}$ in (20) is $\delta \mathbf{K}^{-1} \mathbf{G}(\mathbf{x}, \tau)$, and thus the first-order contribution is:

$$
\mathbf{\Xi}_{1}=\mathbf{h}\left(\varepsilon \mathbf{K}^{-1}(\xi) \mathbf{G}(\xi, \tau)\right)+\langle\mathbf{X}(\xi, \tau)\rangle .
$$

Here the first term is small, and if the average $\langle\mathbf{X}(\xi, \tau)\rangle=0$ (as in Section 2.2), the firstorder contribution does not give us any additional information. The second-order contribution can be calculated to (cf. (14)):

$$
\mathbf{\Xi}_{2}=\left\langle\left.\frac{\partial \mathbf{X}}{\partial \mathbf{x}}\right|_{\mathbf{x}=\xi} \mathbf{u}+\left.\frac{\partial \mathbf{h}}{\partial \mathbf{y}}\right|_{\mathbf{y}=0} \mathbf{K}^{-1}(\xi) \mathbf{G}(\xi, \tau)\right\rangle \text {, where } \mathbf{u}=\int_{\tau_{0}}^{\tau} \mathbf{X}(\xi, \theta) d \theta .
$$

As $\varepsilon \rightarrow 0$, then $\xi \rightarrow \mathbf{x}+O\left(\varepsilon^{2}\right)$ and $\boldsymbol{\eta} \rightarrow \mathbf{y}+O\left(\varepsilon^{2}\right)$ on a time scale $O(1 / \ln \varepsilon)<\tau<O(1 / \varepsilon)(c f$. (15)).

Equations (20)-(23) generalize the approach of calculating approximate equations of slow motion, for systems with several strongly damped state variables.

\section{Application example: Failure of standard optimal control under HF excitation}

\subsection{The problem}

Here we employ the general results (20)-(23) to illustrate how a classical control system, which is inherently strongly damped, in the presence of $\mathrm{HF}$ excitation may behave quite differently than expected.

Fig. 5 shows the system, which is often used to test or illustrate control algorithms [13]. It contains a cart used to balance a pendulum in the up-pointing position, against gravity. The system state is defined by the pendulum angle $\theta$ and the cart position $S$, as observed from the rigid platform. The cart has mass $M$ and linear damping coefficient $d$, and the pendulum has mass $m$ and rotational inertia $J$ about its center of gravity at distance $L$ from the loss-free hinge. The rigid platform can be vibrated relative to a fixed inertial frame, with horizontal and vertical amplitude $\bar{a}_{h}$ and $\bar{a}_{v}$, respectively, and frequency $\Omega$. The system reaction to perturbations is governed by a feedback control force, $U=U(S, \dot{S}, \theta, \dot{\theta})$, imposed horizontally to the cart. M otions of this system are governed by: 


$$
\begin{aligned}
& \ddot{s}+2 \beta \dot{s}+\alpha\left(\ddot{\theta} \cos \theta-\dot{\theta}^{2} \sin \theta\right)=u+a_{h} \omega^{2} \sin (\omega t+\gamma) \\
& \ddot{\theta}-\left(1-a_{v} \omega^{2} \sin \omega t\right) \sin \theta+\ddot{s} \cos \theta=a_{h} \omega^{2} \sin (\omega t+\gamma) \cos \theta,
\end{aligned}
$$

where non-dimensional variables and parameters are given by (cf. Fig. 5):

$$
\begin{aligned}
& \omega_{0}=\sqrt{\frac{g}{l}} ; t=\omega_{0} \tilde{t} ; \quad l=L\left(1+\frac{J}{m L^{2}}\right) ; \quad u=\frac{U}{(M+m) \omega_{0}^{2} l} ; \omega=\frac{\Omega}{\omega_{0}} \\
& s=\frac{S}{l} ; \quad a_{h}=\frac{\bar{a}_{h}}{l} ; \quad a_{v}=\frac{\bar{a}_{v}}{l} ; \alpha=\frac{m L}{(M+m) l}<1 ; \quad \beta=\frac{d}{2 M \omega_{0}} .
\end{aligned}
$$

The control $u$ in (24) is intended to keep the system at zero state, $\mathbf{z}=\left\{\begin{array}{llll}s & \dot{s} & \theta & \dot{\theta}\end{array}\right\}^{T}=\mathbf{0}$, and return it to zero in case of any perturbations due to initial conditions or external forces. It is designed as an optimal linear regulator, basing its actions on a linearized model of the unforced system to be controlled, and on measurements - assumed here to be error- and noisefree - of instantaneous values of the state $\mathbf{z}$ i.e., $u=u(\mathbf{z})$. The linearized model of the unforced system can be obtained from (24) by letting $a_{v}=a_{h}=0$, and linearising for small values of $\theta$ and $\dot{\theta}$, to obtain a system of linear first-order equations in state space form:

$$
\begin{aligned}
\dot{\mathbf{z}}=\mathbf{A z} & +\mathbf{B} u, \text { where } \\
\mathbf{A} & =\left[\begin{array}{cccc}
0 & 1 & 0 & 0 \\
0 & \frac{-2 \beta}{1-\alpha} & \frac{-\alpha}{1-\alpha} & 0 \\
0 & 0 & 0 & 1 \\
0 & \frac{2 \beta}{1-\alpha} & \frac{1}{1-\alpha} & 0
\end{array}\right] ; \mathbf{B}=\left[\begin{array}{c}
0 \\
\frac{1}{1-\alpha} \\
0 \\
\frac{-1}{1-\alpha}
\end{array}\right] .
\end{aligned}
$$

The control law $u(\mathbf{z})$ for the optimal linear state-feedback controller is:

$$
u=-\hat{\mathbf{k}}^{T} \mathbf{z} .
$$

where the optimal gain vector $\hat{\mathbf{k}}$ is chosen so as to minimize a weighted sum of integrated control error and control effort:

$$
\int_{0}^{\infty}\left(\mathbf{z}^{T} \mathbf{Q} \mathbf{z}+R u(\mathbf{z})^{2}\right) d t
$$

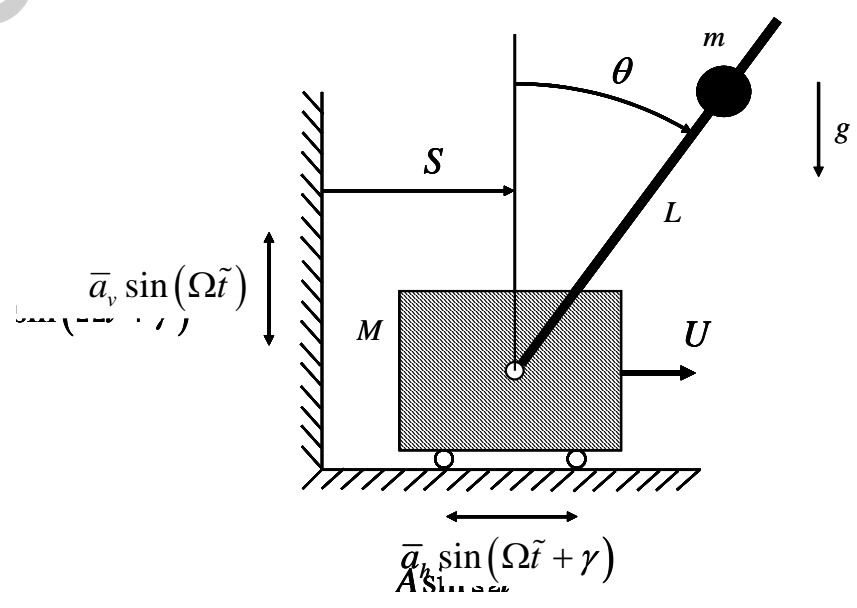

Fig. 5. Inverted pendulum, balanced by a moving cart. 
where $\mathbf{Q}$ and $R$ are positive definite weighting factors. These factors are chosen to reflect a suitable trade-off between the conflicting requirements of quickly reducing the setpoint error to a small value (large $\mathbf{Q} / R$ ), without expending excessively large control power (small $\mathbf{Q} / R$ ). The minimizing solution for $\mathbf{k}$ can be found in many texts on control theory (e.g. [13]):

$$
\hat{\mathbf{k}}=R^{-1} \mathbf{B}^{T} \mathbf{S},
$$

where $\mathbf{S}$ is the solution of the algebraic Riccati equation:

$$
\mathbf{A}^{T} \mathbf{S}+\mathbf{S A}-R^{-1} \mathbf{S B B}^{T} \mathbf{S}+\mathbf{Q}=0 .
$$

For given values of $(\mathbf{A}, \mathbf{B}, \mathbf{Q}, R)$, the Riccati matrix $\mathbf{S}$ is readily computed using, e.g., the builtfunction LQR in MATLAB [14] .

Thus, for a given mass ratio $\alpha$ and damping $\beta$ of the physical system, $\mathbf{A}$ and $\mathbf{B}$ is calculated using (26), and with chosen $\mathbf{Q}$ and $R$, the Riccati matrix is given by the solution to (30). Then $\hat{\mathbf{k}}$ is calculated using (29), and the control force $u$ can be calculated using (27) for any state $\mathbf{z}$, i.e. in explicit form:

$$
u=-\hat{k}_{1} s-\hat{k}_{2} \dot{s}-\hat{k}_{3} \theta-\hat{k}_{4} \dot{\theta} .
$$

where the control coefficients $\hat{k}_{1-4}$ are typically large, compared to the parameters of the uncontrolled part of the system (26).

Near the setpoint $\mathbf{z}=\mathbf{0}$, the controlled system is governed by (26) with (27) inserted, i.e. $\dot{\mathbf{z}}=\overline{\mathbf{A}} \mathbf{z}$ where $\overline{\mathbf{A}}=\mathbf{A}-\mathbf{B} \hat{\mathbf{k}}^{T}$. The optimal control (29) with (30) then guaranties that all eigenvalues of $\overline{\mathbf{A}}$ have negative real parts, so that the setpoint is asymptotically stable to small perturbations.

\subsection{System response with strong HF excitation: Numerical simulation}

The question is how the controlled system behave in the presence of small but rapid oscillations of the supporting platform, i.e. if $0<a_{h} \square 1,0<a_{v} \square 1$, and $\omega \square 1$ in (24). It was already mentioned in Section 2 how elliptic HF excitation may cause equilibrium bias, shifting the equilibrium(s) of the pendulum away from being straight down (or up). The control system, however, might be able to compensate for this just by using more power, so that the performance of the controller could be expected to be only slightly affected by the HFexcitation. The setpoint $\mathbf{z}=\mathbf{0}$ would still remain stable, though with a small overlay of $\mathrm{HF}$ oscillations corresponding to the excitation. Or another stable equilibrium point would appear, deviating less from $\mathbf{z}=\mathbf{0}$ for larger allowable control efforts.

Numerical simulations contradict these expectations. As appears from Fig. 6, the controller works perfectly if there is either no (Fig. 6a), or only horizontal (Fig. 6b), or only vertical (Fig. 6c) HF-excitation, in all cases stabilizing the pendulum in the up-pointing position, and moving the cart to the zero point. The pendulum cart motions are overlaid by small HFoscillations, but on the average the control system fulfills its objective. However, as appears from Fig. 7, it fails when vertical and horizontal oscillations are applied simultaneously. The control system still stabilizes the cart and the pendulum, but the final average cart position is not even close to zero, and the pendulum is stabilized with some permanent tilt, i.e. both state variables are biased (Fig. 7a). An attempt to reduce the permanent error through increased control effort does not help, the stronger controller just amplifies the misbehavior (Fig. 7b). N ext we investigate this effect theoretically. 


\subsection{Asymptotic analysis}

Here we derive and examine an approximate analytic prediction for the biased equilibrium state. A s for the classical analysis in [13] (for the system without HF excitation) we let $\alpha=0$, which corresponds to neglecting the horizontal reaction force between the cart and the pendulum. This restriction, assuming a very light or short pendulum, greatly simplifies the analysis, without sacrificing essential features of the system. We also neglect mechanical damping $\beta$, as compared to the dominating damping coefficient $\hat{k}_{2}$ of the control system. Making allowance for domination of the control terms we assume the corresponding coefficients to be of the same magnitude order as the high excitation frequency, i.e. $\hat{\mathbf{k}}=\omega \mathbf{k}, \mathbf{k}=O(1)$. Taking (24) along with the control force (31) into account we obtain the equations of motion:

$$
\begin{aligned}
& \ddot{s}=-\omega k_{1} s-\omega k_{2} \dot{s}-\omega k_{3} \theta-\omega k_{4} \dot{\theta}+a_{h} \omega^{2} \sin (\omega t+\gamma) \\
& \ddot{\theta}=\omega\left(k_{1} s+k_{2} \dot{s}+k_{3} \theta+k_{4} \dot{\theta}\right) \cos \theta+\left(1-a_{v} \omega^{2} \sin \omega t\right) \sin \theta .
\end{aligned}
$$

In [5] the linear approximation of this system was investigated, while here we apply asymptotic analysis to the full nonlinear system.

To transform (32) to a form suitable for averaging, we first introduce a fast time $\tau=\omega t$ and a small parameter $\varepsilon=\omega^{-1} \square 1$, and add new dependent variables $p(t)$ and $q(t)$ for converting to a system of first-order equations:

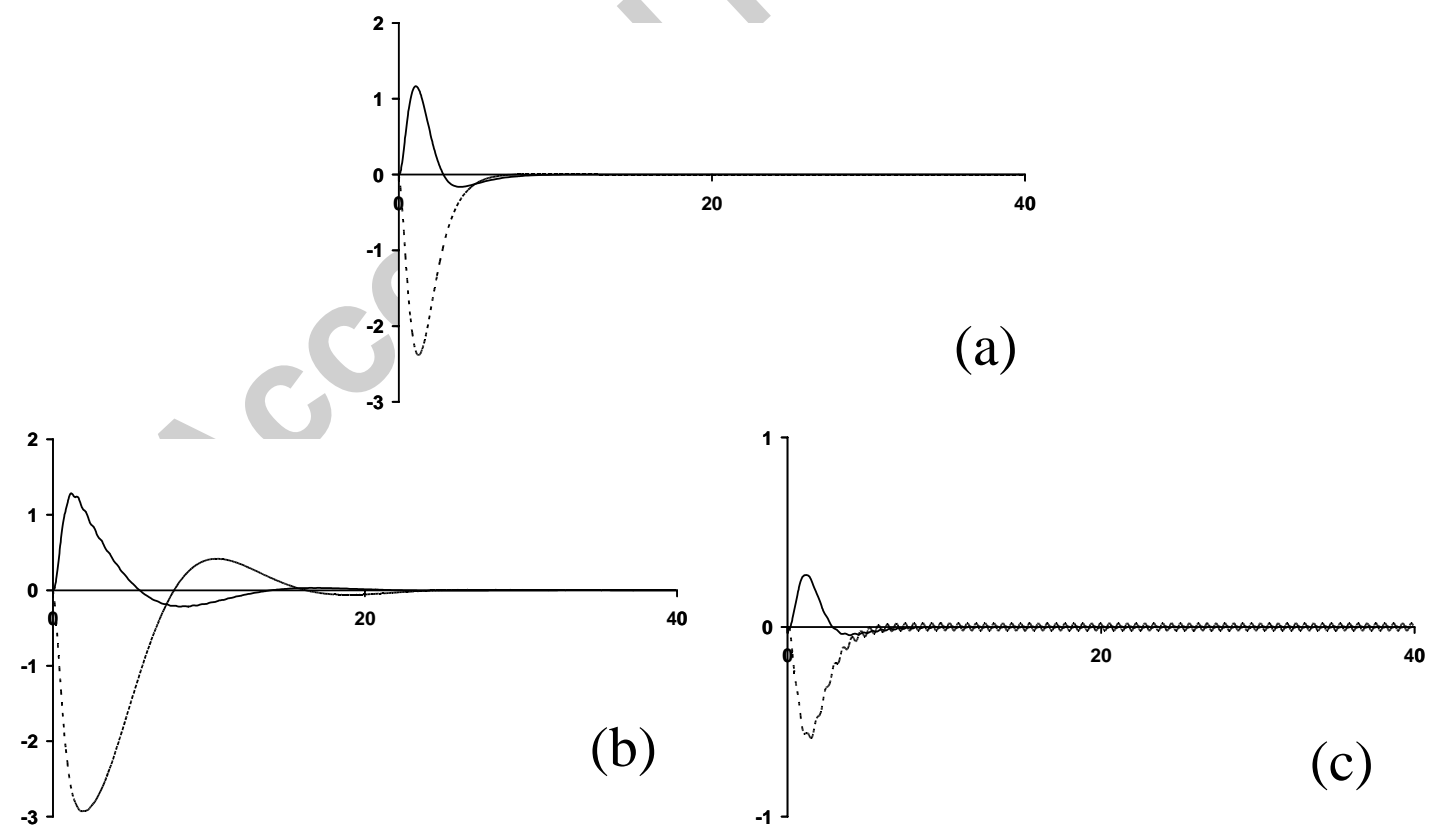

Fig. 6. Results of numerical simulation of the cart and the pendulum system (24) under optimal control (31), when there is (a) no HF-excitation $\left(a_{h}=a_{v}=0\right)$; (b) only horizontal HF-excitation $\left(a_{h}=0.02\right)$; (c) only vertical HF-excitation $\left(a_{v}=0.02\right)$. Solid line: pendulum tilt $\theta(t)$; D otted line: cart position $s(t)$. Parameters: $\alpha=0, \beta=0$,

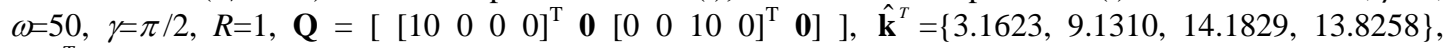
$\mathbf{z}(0)^{T}=\{0,0,0,0.5\}$. 


$$
\begin{aligned}
& s^{\prime}=\varepsilon p \\
& \theta^{\prime}=\varepsilon q \\
& p^{\prime}=-k_{2} p-k_{4} q+A_{h} \sin (\tau+\gamma)-\varepsilon\left(k_{1} s+k_{3} \theta\right) \\
& q^{\prime}=\left(k_{2} p+k_{4} q\right) \cos \theta-A_{v} \sin \theta \sin \tau+\varepsilon\left(\left(k_{1} s+k_{3} \theta\right) \cos \theta+\sin \theta\right) .
\end{aligned}
$$

where ()$^{\prime}=d / d \tau, A_{v}=a_{v} \omega=O(1), A_{h}=a_{h} \omega=O(1)$, and the variables $s$ and $\theta$ are seen to be slowly varying in $\tau$. Next we eliminate the large oscillating terms in the equations for $p$ and $q$, by introducing a third slow variable, next to $s$ and $\theta$. This can be achieved by calculating first the particular solution of the unperturbed system ((33) with $\varepsilon=0)$, which gives that $\theta$ and $s$ are constants, and a third equation (obtained by computing $p^{\prime} \cos \theta+q^{\prime}$ for $\varepsilon=0$, which can be integrated with respect to $\tau$ to give:

$$
x=p \cos \theta+q+A_{h} \cos \theta \cos (\tau+\gamma)-A_{r} \sin \theta \cos \tau .
$$

where $x$ is a constant of integration. Then consider instead (34) as the definition of a new dependent variable $x(\tau)$. Eliminating $q$, the corresponding transformed form of (33) becomes:

$$
\begin{aligned}
s^{\prime}= & \varepsilon p \\
\theta^{\prime}= & \varepsilon\left(x-p \cos \theta-A_{h} \cos \theta \cos (\tau+\gamma)+A_{v} \sin \theta \cos \tau\right) \\
x^{\prime}= & \varepsilon \sin \theta-\theta^{\prime}\left(p \sin \theta+A_{h} \sin \theta \cos (\tau+\gamma)+A_{r} \cos \theta \cos \tau\right) \\
p^{\prime}= & -\left(k_{2}-k_{4} \cos \theta\right) p-k_{4}\left(x-A_{h} \cos \theta \cos (\tau+\gamma)+A_{v} \sin \theta \cos \tau\right) \\
& +A_{h} \sin (\tau+\gamma)-\varepsilon\left(k_{1} s+k_{3} \theta\right) .
\end{aligned}
$$

The first three equations now have the standard form, i.e. their right-hand sides are small, but the last one contains non-small oscillating terms (those with $A_{h}, A_{v}$ ), as well as non-small linear terms (those with $k_{2}$ and $k_{4}$ ) expressing strong damping due to optimal control. The oscillating terms can be eliminated if we apply the following transformation based on the particular periodic solution to the unperturbed system:
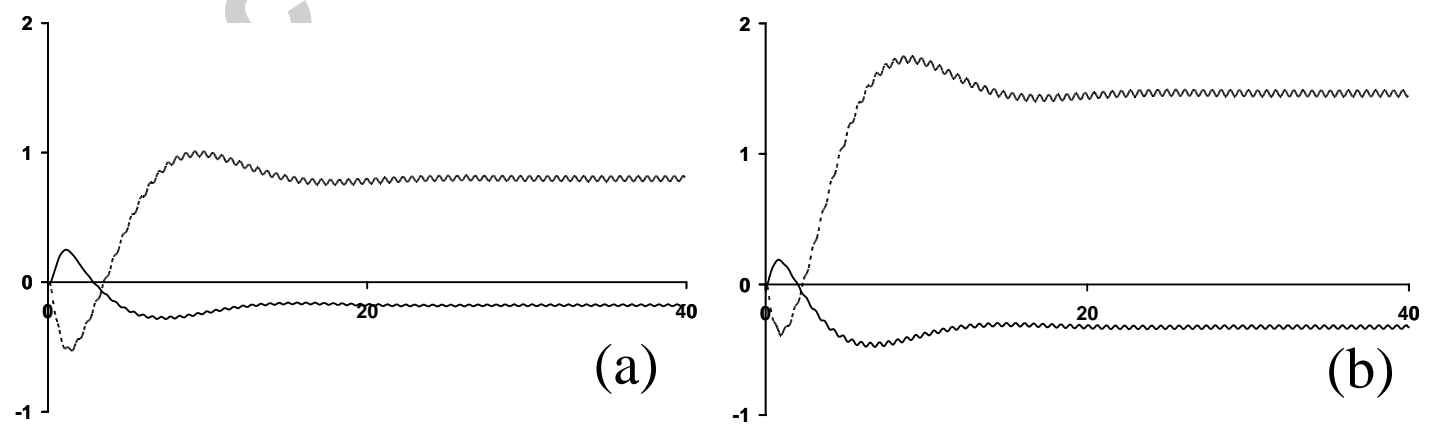

Fig. 7. Results of numerical simulation of the cart and the pendulum system (24) under optimal control (31), when there is simultaneous horizontal and vertical HF-excitation $\left(a_{h} a_{v} \neq 0\right)$, and (a) controller gain $\hat{\mathbf{k}}^{T}=\{3.1623,9.1310,14.1829,13.8258\}$; (b) stronger (twice the one for (a)) controller gain $\hat{\mathbf{k}}^{T}=\{6.3246$, $18.2620,28.3658,27.6516\}$. Solid line: pendulum tilt $\theta(t)$; Dotted line: cart position $s(t)$. Parameters (unless otherwise stated): as for Fig. 6. 


$$
\begin{aligned}
p=r+\lambda_{1}(\theta)+\lambda_{2}(\theta) \sin \tau+\lambda_{3}(\theta) \cos \tau, \text { where } \\
\lambda_{1}(\theta)=-\frac{k_{4} x}{k_{2}-k_{4} \cos \theta} \\
\lambda_{2}(\theta)=\frac{A_{h}\left(k_{2}\left(\cos \gamma-k_{4} \cos \theta \sin \gamma\right)+\left(1+k_{4}^{2} \cos ^{2} \theta\right) \sin \gamma\right)-k_{4} A_{v} \sin \theta}{1+\left(k_{2}-k_{4} \cos \theta\right)^{2}} \\
\lambda_{3}(\theta)=\frac{A_{h}\left(k_{2}\left(\sin \gamma+k_{4} \cos \theta \cos \gamma\right)-\left(1+k_{4}{ }^{2} \cos ^{2} \theta\right) \cos \gamma\right)}{1+\left(k_{2}-k_{4} \cos \theta\right)^{2}} \\
\quad-\frac{k_{4} A_{v} \sin \theta\left(k_{2}-k_{4} \cos \theta\right)}{1+\left(k_{2}-k_{4} \cos \theta\right)^{2}} .
\end{aligned}
$$

Substituting this into (35), the original system (32) has been transformed into its final form:

$$
\begin{aligned}
s^{\prime}= & \varepsilon\left(r+\lambda_{1}(\theta)+\lambda_{2}(\theta) \sin \tau+\lambda_{3}(\theta) \cos \tau\right) \\
\theta^{\prime}= & \varepsilon\left(x-A_{h} \cos \theta \cos (\tau+\gamma)+A_{2} \sin \theta \cos \tau\right) \\
& -\varepsilon\left(r+\lambda_{1}(\theta)+\lambda_{2}(\theta) \sin \tau+\lambda_{3}(\theta) \cos \tau\right) \cos \theta \\
x^{\prime}= & \varepsilon \sin \theta-\theta^{\prime}\left(A_{h} \sin \theta \cos (\tau+\gamma)+A_{1} \cos \theta \cos \tau\right) \\
& -\theta^{\prime}\left(r+\lambda_{1}(\theta)+\lambda_{2}(\theta) \sin \tau+\lambda_{3}(\theta) \cos \tau\right) \sin \theta \\
r^{\prime}= & -\left(k_{2}-k_{4} \cos \theta\right) r+O(\varepsilon) .
\end{aligned}
$$

Since (37) has the general form (20), the general solution (21)-(23) applies, provided $k_{2}-k_{4}$ is positive. The latter will normally hold true, since $k_{2}-k_{4}>0$ is the necessary condition for stability of the control algorithm in the linear case. The first-order approximation, obtained by retaining only terms to the lowest order of $\varepsilon$ in each equation in (21) becomes:

$$
\begin{aligned}
s_{1}^{\prime} & =\varepsilon \lambda_{1} \\
\theta_{1}^{\prime} & =\varepsilon\left(x_{1}-\lambda_{1} \cos \theta_{1}\right) \\
x_{1}^{\prime} & =\varepsilon \sin \theta_{1}-\varepsilon \lambda_{1}\left(x_{1}-\lambda_{1} \cos \theta_{1}\right)+\frac{1}{2} \varepsilon \cos 2 \theta_{1}\left(A_{h} A_{v} \cos \gamma+A_{v} \lambda_{3}\right) \\
& +\frac{1}{2} \varepsilon \sin 2 \theta_{1}\left(\frac{1}{2}\left(A_{h}^{2}-A_{v}^{2}\right)+\frac{1}{2}\left(\lambda_{3}^{2}+\lambda_{2}^{2}\right)+A_{h}\left(\lambda_{3} \cos \gamma-A_{h} \lambda_{2} \sin \gamma\right)\right) \\
r_{1}^{\prime}= & -\left(k_{2}-k_{4} \cos \theta_{1}\right) r_{1} .
\end{aligned}
$$

This approximation determines the stationary tilt of the pendulum, however it is insufficient for finding the position of the cart. As a matter of fact, the corresponding stationary solutions can be readily obtained by equating to zero the left-hand sides, and solving the algebraic equations for $\left(s_{1}, \theta_{1}, x_{1}, r_{1}\right)$. Substituting the expression for $\lambda_{1}$ from (36), one finds that the first, second, and fourth equation just yield trivial stationary solutions $x_{1}=0, r_{1}=0$, while $s_{1} \in R$ (to first order of approximation). Thus the important equation is the third one. Substituting into this equation $\lambda_{2}$ and $\lambda_{3}$ from (36), linearising near $\theta_{1}=0$, and letting $x_{1}^{\prime}=0$, we obtain an approximate prediction for the stationary tilt $\theta_{1}=\theta_{1 s t}$ of the pendulum: 


$$
\theta_{1 s t}=\frac{A_{h} A_{v} k_{2}\left(\sin \gamma-\left(k_{2}-k_{4}\right) \cos \gamma\right)}{2\left(1+\left(k_{2}-k_{4}\right)^{2}\right)+A_{h}{ }^{2} k_{2}^{2}-A_{v}^{2}\left(1+k_{2}\left(k_{2}-k_{4}\right)\right)} .
$$

As mentioned, the first-order approximation (38) does not give information about the stationary value of cart position $s_{1}$. The second-order approximation is needed for that, as for the uncontrolled pendulum in Section 2.2. This is obtained by including all terms in (21), and calculating the corresponding stationary value of $s_{1}$. O mitting for brevity these elaborate calculations, we state here the final and quite simple result:

$$
\theta_{2 s t}=\theta_{1 s t}, s_{2 s t}=-\frac{k_{3}}{k_{1}} \theta_{2 s t},
$$

according to which the controller will stabilize the cart at a position, whose offset from zero is proportional to the stationary pendulum tilt.

Equation (39) can be also easily transformed into dimensional form:

$$
\begin{gathered}
\theta_{1 s t}=\frac{\bar{a}_{h} \bar{a}_{v} \hat{k}_{2} \Omega^{3}\left(\frac{\sin \gamma}{\sqrt{\frac{L}{g}\left(1+\frac{J}{m L^{2}}\right)}}-\left(\hat{k}_{2}-\hat{k}_{4}\right) \Omega \cos \gamma\right.}{G-\frac{\bar{a}_{v}^{2} \Omega^{2} g}{L\left(1+\frac{J}{m L^{2}}\right)}+\Omega^{4}\left(\bar{a}_{h}^{2} \hat{k}_{2}^{2}-\bar{a}_{v}^{2} \hat{k}_{2}\left(\hat{k}_{2}-\hat{k}_{4}\right)\right)} \\
G=2 g^{2}+2 g\left(\hat{k}_{2}-\hat{k}_{4}\right)^{2} L\left(1+\frac{J}{m L^{2}}\right)
\end{gathered}
$$

Here $G$ holds the terms that are independent of vibration amplitudes. In the important case of small gravity we obtain the following approximation:

$$
\theta_{1 s t}=-\frac{\bar{a}_{h} \bar{a}_{v}\left(\hat{k}_{2}-\hat{k}_{4}\right) \cos \gamma}{\bar{a}_{h}^{2} \hat{k}_{2}-\bar{a}_{v}^{2}\left(\hat{k}_{2}-\hat{k}_{4}\right)}, g<L \Omega^{2}
$$

This form of the solution demonstrates that if the vibration acceleration is dominant compared to gravity, i.e. $\tilde{\omega}^{2}>>g / l$, then the stationary behavior of the damped pendulum is similar to that discussed in section 2.2 .

\subsection{Comments to the theoretically predicted equilibrium state}

The prediction (39)-(40) of the stationary state is identical to what was obtained in [3] by the method of direct separation of motions. However, it differs from the result in $[5,11]$, which was obtained for a linearization of a system similar to (32), and lacks the middle term in the denominator of (39). This deviation is yet a demonstration, beyond what was observed al ready in e.g. [2,8], of the significance of the choice of linearization stage when calculating effects of HF-excitation for nonlinear systems: Results obtained for a linearized system may differ from linearized results of a nonlinear analysis.

The structure of the approximate prediction (39) is similar to that of a simple strongly damped pendulum (18). As appears from (39)-(40), a nonzero value for $\theta_{1 s t}$ and $s_{2 s t}$ can occur only when $A_{h} A_{v} \neq 0$, i.e. when the $\mathrm{HF}$ excitation is not purely horizontal or vertical. 
Further, (40) reveals that if $k_{3} / k_{1}>0$ then $s_{2 s t} / \theta_{s t}<0$, that is: with the controller adding positive (or negative) stiffness to both the cart and the pendulum part of the system, the cart and the pendulum will be biased in opposite directions. This is a consequence of the function (28) to be minimized by the optimal controller, including the choice of weighting constants $\mathbf{Q}$ and $R$. It is unrelated to the $\mathrm{HF}$ excitation, but merely reflects that at equilibrium $(\dot{s}=\dot{\theta}=0)$, the function (28) will be minimal for $u(\mathbf{z})^{2}=0$, which implies $\left(k_{1} s+k_{3} \theta\right)^{2}=0$ and thus (40).

It also appears from (39)-(40) that the phase difference $\gamma$ between vertical and horizontal excitation can be used to control the equilibrium bias. At a certain phase difference, $\gamma=\arctan \left(\left(k_{4}-k_{2}\right) / \omega\right)$, the up-pointing, unbiased pendulum equilibrium $\theta=0$ even becomes stable, as if the system were not HF-excited at all.

Finally we note from (39) that the main source of bias is the part of the controller which damps oscillations of the cart: If $k_{2}=0$, i.e. the controller doesn't damp cart motions (cf. (31)), then the right-hand side of (39) vanishes, and $\theta_{1 s t}=0$ becomes a stationary, unbiased equilibrium.

\subsection{Comparison with numerical simulation}

Figs. 8 and 9 compare results of the approximate analytical prediction (39), (40) to results of numerical simulation of the original equations (32). MATLAB's built-in function ODE45 [14] was used for the numerical simulation, though other integrators were tested with similar results. The simulations were run until stationary conditions were attained, whereafter average values and oscillation amplitudes were calculated numerically from the stationary part of the time series.

Fig. 8 shows a prediction given by (39), (40) of how the stationary average value of the

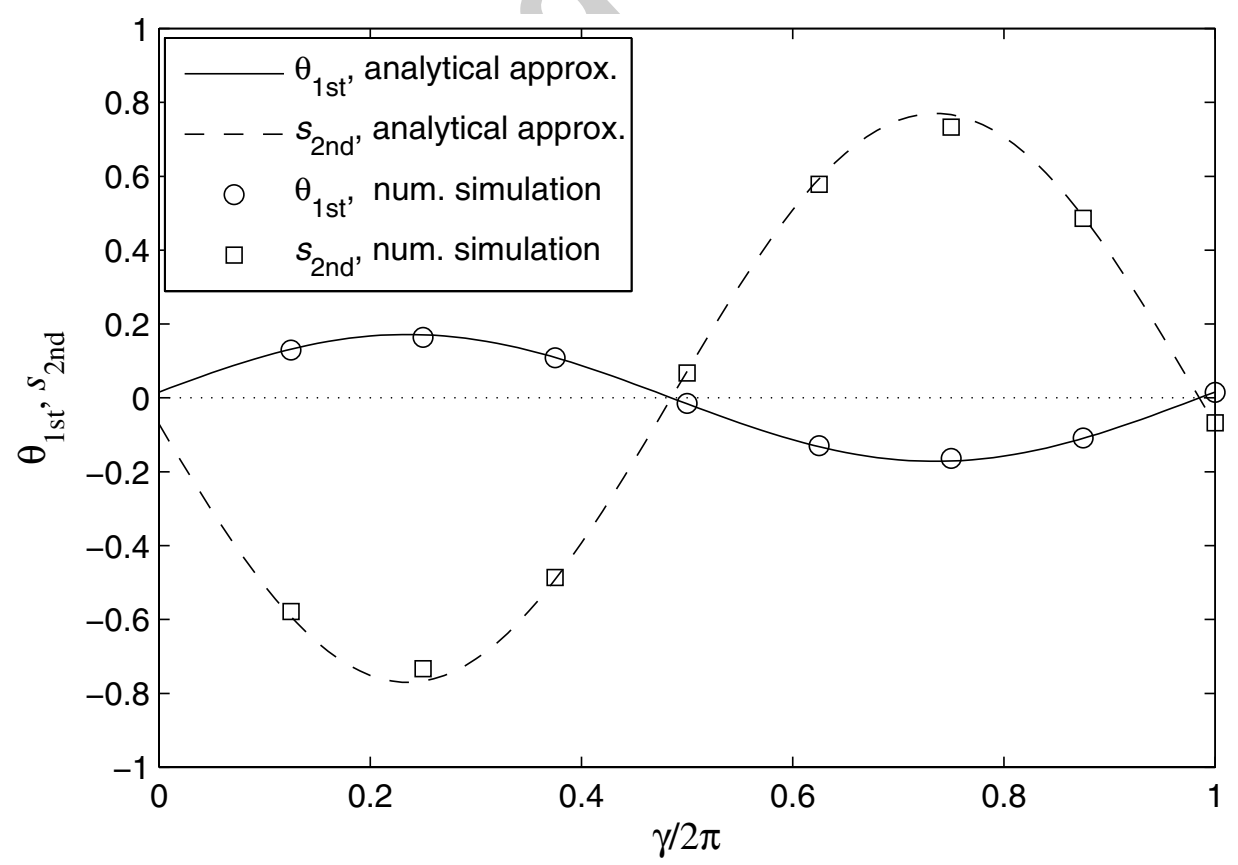

Fig. 8. Stationary average pendulum angle $\theta_{1 s t}$ and cart position $s_{2 \text { nd }}$ of the optimally controlled inverted pendulum, as a function of the phase difference $\gamma$ between horizontal and vertical HF excitation. Parameters: $\alpha=\beta=0$, $\left.\left.R=1, \mathbf{Q}=\left[\begin{array}{llll}10 & 0 & 0 & 0\end{array}\right]^{\top} \mathbf{0}\left[\begin{array}{llll}0 & 0 & 10 & 0\end{array}\right]^{\top} \mathbf{0}\right]\right], \mathbf{k}=\{3.1623 ; 9.1310 ; 14.1829 ; 13.8258\}^{\top}, \omega=50, a_{h}=a_{v}=0.02$. 
pendulum angle $\theta$ (solid line) and cart position $s$ (dashed) varies with the phase difference $\gamma$ of the HF excitation. The agreement between the analytical predictions and numerical simulation (in marker symbols) is seen to be very good. As appears a substantial bias - an offset from the control setpoint at zero - occurs when the phase differences is near $\pi / 2$ or $3 \pi / 2$, where the HF excitation translates the platform along a circular pattern (for $a_{h}=a_{v}$ ). The bias diminishes when the phase difference is near integer multiples of $\pi$ where the platform translates along straight lines.

Fig. 9 shows how the bias increases with increasing vertical HF excitation amplitude. With increased bias, it appears, the agreement between analytical prediction and numerical simulation becomes poorer, reflecting that (39) is valid only for small values of the pendulum average angle. A ctually (39) predicts that the bias approaches infinity, reverses sign, and then drops off for even higher values of $A_{v}$. For the "real" system, however, numerical simulation shows that the bias just increases monotonically.

\subsection{Comments on the chosen asymptotic approach}

The chosen asymptotic approach, based on the modified averaging procedure for systems with several strongly damped state variables, is definitely not the only one suitable for the considered problem. The same results can be obtained, for example, by the method of direct separation of motions $[1,3,5]$. However, it is supposed that for strongly damped systems it is important to distinguish between master and slave variables (cf. (13)), which was underlined by the chosen asymptotic procedure.

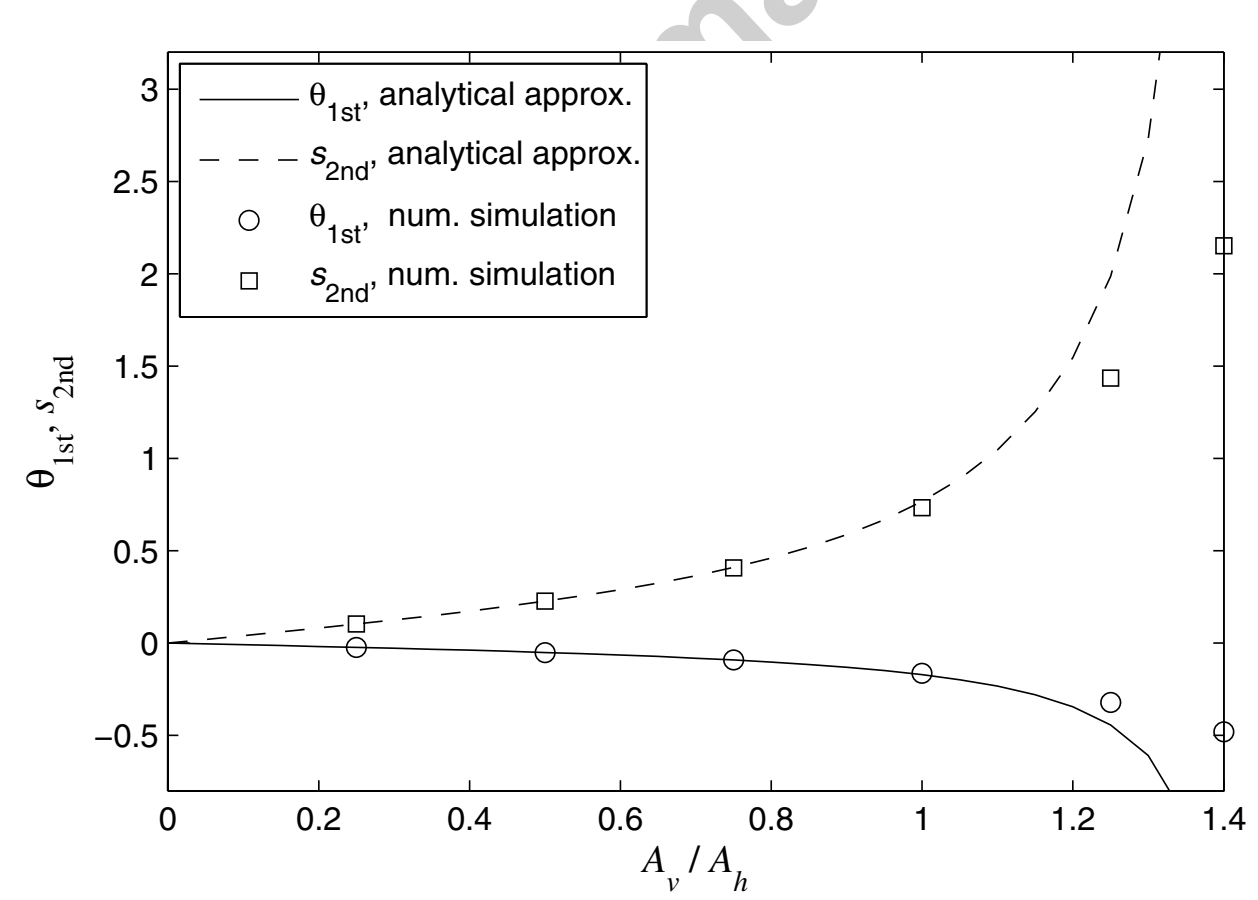

Fig. 9. Stationary average pendulum angle $\theta_{1 \text { st }}$ and cart position $s_{2 \text { nd }}$ of the optimally controlled inverted penduIum, as a function of the HF excitation amplitude ratio $A_{v} / A_{h}$, with $A_{h}$ kept constant. Parameters: $\gamma=\pi / 2$, and otherwise as for Fig. 8 . 


\section{Conclusions}

According to the theoretical predictions of this work, strong high-frequency excitation may change the equilibria of strongly damped systems in a manner that differs substantially from that which is well known for slightly damped systems. The analysis of this has been illustrated above for a simple example, using a slightly modified averaging approach. This has been generalized to a broader class of systems, and exemplified for a classical control problem. Numerical simulations were shown to support the theoretical predictions, and thus a natural next step is to test the predictions with a simple laboratory setup.

The effects of HF excitation for strongly damped systems, investigated in this paper, may be important at least in two areas: Control science, and microsystems.

In control science HF excitation has been known and used for a long time in the form of the so-called dither signals, for example, for smearing out discontinuities such as may be associated with dry friction and sticking behavior [15]. However, with strong damping terms, quite usual in linear feedback control system in general, the HF excitation can cause unexpected bias, like in the example investigated in Section 4. The problem can be also solved in the framework of the control science for example by the appropriate filtering of the velocity signal (cf. [5]).

For microsystems the effects of $\mathrm{HF}$ excitation is relevant, and maybe important, because of the increasing role of damping with downsizing. The authors expect that HF effects may be a key for explaining several experimental observations of effects of microwave radiation on large molecules (like proteins) and nano-structures in solvents [16]. The corresponding model equations are much more complex than for the present study, and typically contain integral terms describing long-term interaction of the micro-object with the fluid. The investigation of such effects, and the development of the appropriate mathematical approaches, could be an important direction for future work.

\section{References}

[1] Blekhman I. I., Vibrational Mechanics - Nonlinear Dynamic Effects, General Approach, Applications, World Scientific, Singapore, 2000.

[2] Thomsen J. J., Some general effects of strong high-frequency excitation: stiffening, biasing, and smoothening, Journal of Sound and Vibration, 253 (2002) 807-831.

[3] Thomsen J. J., Slow high-frequency effects in mechanics: problems, solutions, potentials, International Journal of Bifurcation and Chaos 15 (2005) 2799-2818.

[4] Bogoliubov N. N. and M itropolskii Y u. A., Asymptotic methods in the theory of nonlinear oscillations, Gordon and B reach, N ew Y ork, 1961.

[5] Fidlin A., Nonlinear Oscillations in Mechanical Engineering, Springer, Berlin - Heidelberg, New Y ork, 2006.

[6] Nayfeh A. H., Perturbation Methods, W iley Interscience, N ew Y ork, 1973.

[7] Blekhman I. I. (Ed), Selected Topics in Vibrational Mechanics, Series A on Stability, Vibration and Control of Systems, V olume 11, W orld Scientific, Singapore, 2004.

[8] Thomsen J. J., Vibrations and Stability: Advanced Theory, Analysis and Tools (2 ${ }^{\text {nd }}$ ed.), Springer-V erlag, Berlin - Heidel berg, 2003.

[9] Kovaleva A. S., Optimal Control of Mechanical Oscillations, Springer-V erlag, B erlin - Heidelberg, 1999.

[10] Bullo F., A veraging and Vibrational Control of M echanical Systems, SIAM Journal on Control 
and Optimization, 41 (2002) 542-562.

[11] Fidlin A . and Thomsen J. J., Non trivial effect of strong high-frequency excitation on a nonlinear controlled System, in ICTAM2004 Abstract Book and CD-ROM Proceedings (XXI Int. Congress of Theoretical and Applied mechanics, Warsaw, 15-21 A ugust, 2004), eds. Gutkowski, W. and K owalewski, T. A., IPPT PA N, Warsaw, 2 pp.

[12] Volosov V. M. and M orgunov B. I., Method of Averaging in the Theory of Nonlinear Oscillating systems, M oscow State Univ., M oscow, 1971 (in Russian).

[13] K wakernaak H. and Sivan R., Linear Optimal Control Systems, W iley Interscience, New Y ork, 1972.

[14] Biran A . and B reiner M ., MATLAB 6 for Engineers, Prentice Hall, L ondon, 2002.

[15] M eerkov S. M., Principle of vibrational control: theory and applications, IEEE Transactions on Automatic Control A C-25 (1980) 755-762.

[16] Copty A. B., Neve-Oz Y., Barak I., Golosovsky M., and Davidov D., Evidence for a specific microwave radiation effect on the green fluorescent protein, Biophysical Journal, 91 (2006) 1413-1423. 